\title{
Diagnostic Value of CT Angiography (CTA) in Carotid Cavernous Fistula (CCF) Patients Using the Digital Subtraction Angiography (DSA) as The Gold Standard: Observation in Dr. Soetomo General Hospital Surabaya from January 2016 to July 2020
}

\section{Laila Amalia ${ }^{1}$, Hartono Yudi Sarastika ${ }^{2}$, Bambang Soeprijanto ${ }^{3}$}

${ }^{1}$ Radiology Resident, Department of Radiology, Faculty of Medicine, Universitas Airlangga, Surabaya, Indonesia ${ }^{2}$ Radiology Staff, Department of Radiology, Faculty of Medicine, Universitas Airlangga, Surabaya, Indonesia ${ }^{3}$ Radiology Staff, Department of Radiology, Faculty of Medicine, Universitas Airlangga, Surabaya, Indonesia

\section{A R T I C L E I N F O Keywords: \\ CT Angiography (CTA) Carotid-Cavernous Fistula $(\mathrm{CCF})$ Internal Carotid Artery (ICA) Direct Indirect \\ *Corresponding author: \\ Hartono Yudi Sarastika \\ E-mail address: \\ hystika@gmail.com} Digital Subtraction Angiography (DSA)

All authors have reviewed and approved the final version of the manuscript.

\section{https://doi.org/10.32539/bsm.v5i6.318}

A B S T R A C T

Background: $\mathrm{CT}$ angiography (CTA) is the initial modality for diagnosing the Carotid Cavernous Fistula ( $\mathrm{CCF}$ ), identifying the type of $\mathrm{CCF}$, measuring the diameter of the fistula and the diameter of the internal carotid artery (ICA) pre and post fistula, and then confirmed by Digital Subtraction Angiography (DSA) as the gold standard. CTA is expected to provide more information to improve the accuracy of CCF diagnosis to benefit therapy and prevent complications. This study aimed to determine the diagnostic value of CTA in CCF patients in Dr. Soetomo General Hospital Surabaya, using the DSA as a gold standard. Methods: An observational retrospective study of $\mathrm{CCF}$ patients met the inclusion and exclusion criteria and underwent CTA and DSA examinations at the Radiology Installation of Dr. Soetomo General Hospital, Surabaya, from January 2016 to July 2020. Results: In the comparison of types of CCF CTA to DSA, the direct group was $53.8 \%$ and $57.7 \%$, respectively, while the indirect group was $46.1 \%$ and $42.3 \%$ with a very strong correlation $(\mathrm{k}=0.922 ; \mathrm{p}=0.000)$, sensitivity was $93.3 \%$, specificity was $100 \%$, PPV of $100 \%$, NPV of $91.7 \%$ and accuracy of $96.15 \%$. Direct type evaluation on the DSA showed a large picture of the fistula diameter; the ICA pre fistula's size was enlarged; therefore, the ICA post fistula diameter was reduced or absent. There was a strong correlation of fistula diameter measurement results between CTA and DSA $(r=0.695, p=0.006)$. Conclusion: CTA has a similarity rate of $92.2 \%$ with DSA in identifying the type of CCF. The accuracy value of CTA is close to DSA in fistula diameter measurements.

\section{Introduction}

The carotid-cavernous fistula $(\mathrm{CCF})$ is a pathological shunt between the carotid artery and the cavernous sinus or directly to the ophthalmic vein. This shunt flow may be a direct fistula or a dural connection between the external carotid artery or internal carotid artery and the cavernous sinus. ${ }^{1}$ The presentation can be varied and nonspecific; however, patients with CCF commonly present with ophthalmic manifestations due to venous drainage of the orbit from the cavernous sinus. ${ }^{2}$

$\mathrm{CCF}$ is a rare entity that can develop spontaneously or due to secondary trauma. ${ }^{2}$ Traumatic CCF account for $75 \%$ of $\mathrm{CCF}$ overall. Previous studies reported $\mathrm{CCF}$ incidence in $0.2 \%$ of patients with craniocerebral trauma and up to $4 \%$ of patients with basilar skull fractures. ${ }^{3}$ Based on the 2018 Basic Health Research 
in Indonesia, the proportion of head injuries in the province of East Java was $11.9 \%$, and CCF incidence in East Java was around $0.0022 \% .4$ According to the demographics of traumatic injuries, traumatic CCF is most commonly seen in young male patients.

Radiological examination is an essential investigation for CCF diagnosis, using ultrasonography (USG), CTA, magnetic resonance angiography (MRA), and DSA modalities. Computed Tomography Angiography (CTA) is a combination of $\mathrm{X}$-rays and computerization that produces imaging in axial slices to display tomographic images that contain more detailed information about the blood vessels. ${ }^{5}$ CTA is a non-invasive first-line imaging modality to evaluate suspected $\mathrm{CCF}$ and identify the presence of trauma such as a fracture closely associated with CCF cases. CTA has advantages, namely the same accuracy as DSA, fast data acquisition, and non-invasive. However, the drawbacks of CTA are highly dependent on contrast agents, radiation exposure and is heavily influenced by the movement. 6,7

Digital Subtraction Angiography (DSA) uses an xray modality to visualized blood flow in blood vessels by injecting a contrast dye then imaging the blood vessels using fluoroscopic techniques. 8 The advantages of DSA are high spatial resolution, faster diagnosis of abnormalities, and the gold standard in therapeutic management. However, DSA also has disadvantages such as invasive procedures, sometimes unable or difficult to determine the size and location of fistula precisely, highly dependent on contrast agents, and prolonged radiation exposure. 6,7

Langer et al. (2004) reported a high-flow variant of CCF with 100\% CTA sensitivity and had a high correlation with angiography to visualize the extent of the lesion, presence of cortical-venous drainage, and the location and morphology of the nidus. If the flow nidus is challenging to identify in low-flow fistulas, secondary signs appeared on CTA, followed by angiography for confirmation diagnostics. The CTA examination is sufficiently accurate and sensitive to describe high-flow cavernous sinus lesions and the usual indirect signs of low flow lesions. 9 In another study, the diagnostic value of CTA did not differ significantly from DSA, and the sensitivity was $87 \%$ and $94.4 \%$, respectively. Therefore, CTA is a relatively sensitive and non-invasive diagnostic test prior to DSA examination. DSA remains the gold standard imaging modality in the vascular examination, diagnosis the classification of CCF type, diagnostic and therapeutic. 10

Besides as a definitive diagnostic of CCF, DSA examination can be evaluated hemodynamically. If there is a large fistula, the blood flow to the fistula will increase. The researchers suspect that the larger the fistula diameter, the smaller the ICA diameter post fistula and the diameter of the ICA pre fistula will be enlarged. CTA helps clinicians evaluate CCF patients because the DSA examination requires time to prepare the embolism material for therapy according to the type of CCF previously evaluated from the CTA examination. The embolic materials included a detachable balloon, detachable coils, polyvinyl acetate (PVA) glue.

There is no data regarding $\mathrm{CCF}$ and CTA examinations associated with the DSA examination at Dr. Soetomo General Hospital, Surabaya. These data are essential to determine any differences from previous studies. Data are expected to be a reference in diagnosis and identifying the type of CCF on the CTA examination and then confirmed by DSA. This study evaluated the diagnostic value of CTA with the gold standard DSA in CCF patients based on direct and indirect type variables, fistula diameter, and pre and post-fistula ICA diameter. There has not been any literature or previous research on the pre and post fistula ICA diameter in CCF.

\section{Methods}

This study was a retrospective observational analytic study. The study population was CCF patients who had undergone CTA and DSA examinations at the Radiology Department at Dr. Soetomo General Hospital Surabaya between January 2016 and July 2020. The research subjects were recruited by consecutive sampling and met the inclusion and exclusion criteria. The inclusion criteria were imaging findings that were 
eligible for the evaluation and reported to the radiologist during the study period.

This study analyzed the type of CCF based on CTA examination, DSA examination, between CTA and DSA; CCF fistula diameter by CTA examination; ICA diameter pre and post fistula by DSA examination; ICA fistula diameter between CTA and DSA; ICA diameter pre and post CCF fistula on CTA and DSA. The CTA examination used the Multislice CT scan 16 slices with the Siemens Somatom brand and 128 slices CT Scan slices with the Philips Ingenuity core brand, while the DSA used the GE Innova 2100 Cath / Angio system, the Philips Allura Xper FD 20 (Clarity IQ), and the Philips Allura Xper FD 10. (Clarity IQ).

Clinical data were obtained from the medical records of the $\mathrm{CCF}$ patient, and an Interventional Radiologist re-evaluated the imaging data of the CTA patient. These data will be analyzed statistically by testing the diagnostic value of CTA compared to the patient's DSA imaging as the gold standard. Data is displayed descriptively in the table of research results.

\section{Results}

The type of CCF and the ICA diameter on the CTA examination were assessed from the multiplanar evaluation, and the maximum intensity projection (MIP) was then followed with an assessment of the largest ICA diameter. Whereas in DSA, the type of CCF and the largest ICA diameter measurement were evaluated in several views with arterial phase. Based on the age and gender of 26 patients, the mean age was $40.54 \pm 17.346$ years, and the median 41.50 years, the minimum age was 12 years, and the maximum age was 77 years (Table 1).

Based on CTA and DSA examinations, CCF types are classified as direct and indirect types. Table 2 shows the direct and indirect types of CTA examinations were $53.8 \%$ and $46.1 \%$, respectively. DSA examination found the direct CCF type was $57.7 \%$, while the indirect type was $42.3 \%$.

In the DSA examination, based on the age group, the direct types were mostly found in the 21-40 years age group $(66.7 \%)$, while the indirect type was in the
$>41$ years age group (61.5\%) (Figure 1). Based on gender, direct and indirect types were found in 10 males and seven females, respectively (Figure 2).

\section{Comparison of CCF types based on the CTA analysis}

The type of CCF on the CTA examination compared to the DSA included direct and indirect CCFs (Table 3). Analysis of the CCF type showed a sensitivity of $93.3 \%$, specificity of $100 \%$, a positive predictive value of $100 \%$, a negative predictive value of $91.7 \%$, and an accuracy of $96.15 \%$. The correlation between the diagnostic value of CTA and DSA using the percent Chi-Square test showed a significant value $(p$-value $=0.000)$, which means that there was a significant relationship between CTA and DSA. This result was confirmed by the Kappa coefficient (k) of 0.922 (which strongly correlates). It concluded that there was an agreement between CTA and DSA.

\section{Size of the fistula diameter on CTA based on the diagnosis of CCF type using DSA examination}

DSA examinations revealed 15 direct CCF and 11 indirect $\mathrm{CCF}$. Measurement of fistula diameter can only be performed to direct CCF with a fistula diameter range of $1.845-5.293 \mathrm{~mm}$ (Table 4). One sample of indirect $\mathrm{CCF}$ on CTA (false negative) was difficult to evaluate the size of the fistula, then confirmed using DSA showed a direct type with a diameter of $1.955 \mathrm{~mm}$.

\section{Correlation of fistula diameter between CTA and DSA}

The fistula diameter in CTA was 1.845 - $5.293 \mathrm{~mm}$ (3.752 \pm 1.062$)$, while in DSA was 1.758 - $5.488 \mathrm{~mm}$ (3.659 \pm 1.251$)$. Pearson Correlation Test (2-tailed) value of 0.006 showed a significant correlation between CTA and DSA fistula diameter. The r-value of 0.695 (69.5\%) indicates a strong relationship (Table 5)

\section{Percentage difference in fistula diameter of ICA on CTA examination to DSA}

Subsequent calculation of the percentage difference between the diameter of a fistula between the CTA and 
DSA showed a mean value of $15.06 \%$ (SD $\pm 14.98 \%)$ and a median of $9.75 \%$ (Table 7). We are also evaluating the percentage difference in intervals of 11 subjects showed that the difference in $0-20 \%$ of the DSA (Table 7).

\section{Comparison of the diameter of the ICA pre and post}

fistula to the diameter of the ICA fistula in DSA

DSA examinations discovered 15 direct $\mathrm{CCF}$ with fistula diameter 1.758 to $5.488 \mathrm{~mm}$, and a diameter ratio of ICA pre- and post-fistula was $1.210-1.879 \mathrm{~mm}$ (Table 8). Five ICA post fistula diameter cannot be evaluated due to high flow (large diameter fistula), which resulted in the entire flow into the fistula; thus, there is no visible flow in the ICA post fistula (distal ICA).

\section{Correlation between ICA diameter pre and post fistula in CTA and DSA}

Measurements were only performed on 20 subjects. Spearman rho test report $r$ value 0,483 or $4.83 \%$, thus these variables have moderate correlation $(p=0.031)$. $\mathrm{p}<0.05$ stated a statistically significant relationship between the pre and post fistula ICA diameter on CTA and DSA examinations (Table 9).

Table 1. Subject characteristics based on age group and sex

\begin{tabular}{ll}
\hline Characteristics & $\mathbf{n}(\%)$ \\
\hline Age group (years old) & \\
\hline $10-20$ & $4(15.4)$ \\
$21-40$ & $9(34.6)$ \\
$>41$ & $13(50)$ \\
\hline Sex & \\
\hline Male & $14(53.8)$ \\
Female & $12(46.2)$ \\
\hline
\end{tabular}

Table 2. Types of CCF on CTA and CSA examination

\begin{tabular}{lc}
\hline \multicolumn{1}{c}{ Type of CCF } & n (\%) \\
\hline $\begin{array}{l}\text { CTA Examination } \\
\text { Right-sided direct }\end{array}$ & $5(19.2)$ \\
Left-sided direct & $9(34.6)$ \\
Bilateral Indirect & $1(3.8)$ \\
Right-sided Indirect & $5(19.2)$ \\
Left-sided Indirect & $6(23.1)$ \\
\hline
\end{tabular}

CSA Examination 


$\begin{array}{lc}\text { Right-sided direct } & 5(19.2) \\ \text { Left-sided direct } & 10(38.5) \\ \text { Bilateral Indirect } & 1(3.8) \\ \text { Right-sided Indirect } & 4(15.4) \\ \text { Left-sided Indirect } & 6(23)\end{array}$

Total $26(100)$

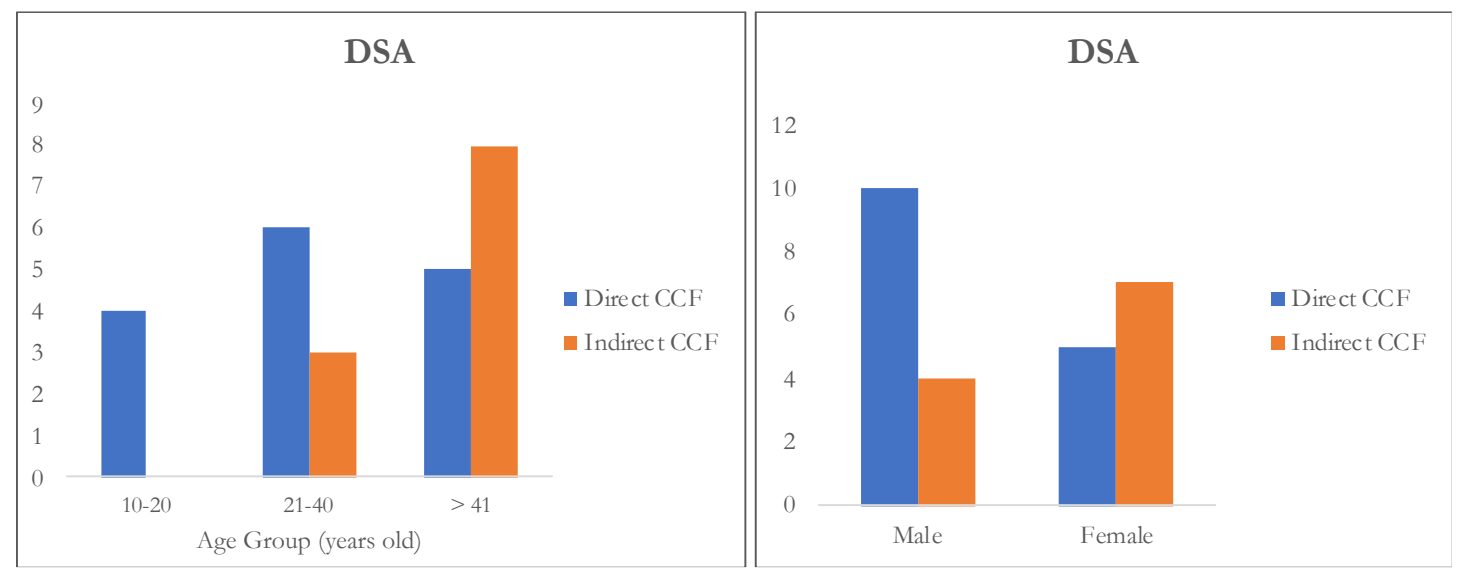

Figure 1. Distribution of CCF type on DSA examination based on age group and sex

Table 3. Analysis of types of CCF based on CTA to DSA

\begin{tabular}{|c|c|c|c|c|c|c|c|c|c|c|}
\hline \multirow{2}{*}{ Variable } & \multirow{2}{*}{$\begin{array}{c}\text { Type of } \\
\text { CCF }\end{array}$} & \multicolumn{2}{|c|}{ DSA } & \multirow{2}{*}{$\mathrm{p}$-value } & \multirow{2}{*}{$\begin{array}{c}\mathrm{r} \\
\text { Kappa }\end{array}$} & \multirow{2}{*}{$\begin{array}{c}\text { Sensitivity } \\
(\%)\end{array}$} & \multirow{2}{*}{$\begin{array}{c}\text { Specificity } \\
(\%)\end{array}$} & \multirow{2}{*}{$\begin{array}{l}\text { PPV } \\
(\%)\end{array}$} & \multirow{2}{*}{$\begin{array}{l}\text { NPV } \\
(\%)\end{array}$} & \multirow{2}{*}{$\begin{array}{c}\text { Accuracy } \\
(\%)\end{array}$} \\
\hline & & Indirect & Direct & & & & & & & \\
\hline & Direct & 14 & 0 & & & & & & & \\
\hline \multicolumn{11}{|l|}{ CTA } \\
\hline & Indirect & 1 & 11 & 0.000 & 0.922 & 93.3 & 100 & 100 & 91.7 & 96.15 \\
\hline Total & & 15 & 11 & & & & & & & \\
\hline
\end{tabular}

Table 4. The size of the fistula diameter using CTA based on the type of CCF in DSA

\begin{tabular}{lr}
\hline Type of CCF & Fistula Diameter using \\
\hline Left-sided direct & 1.845 \\
Left-sided direct & 2.35 \\
Right-sided direct & 2.832
\end{tabular}


Left-sided direct

Right-sided Indirect

Right-sided Indirect

Right-sided Indirect

Left-sided Indirect

Right-sided Indirect

Left-sided Indirect

Left-sided Indirect

Right-sided Indirect

Left-sided Indirect

Left-sided Indirect

Left-sided Indirect

(-) indicates that the fistula diameter size of indirect CCF is difficult to evaluate

Table 5. Pearson Correlation test of fistula diameter measurements for CTA and DSA

\begin{tabular}{|c|c|c|c|c|}
\hline Examination & $\begin{array}{c}\text { Fistula } \\
\text { Diameter (mm) }\end{array}$ & Mean \pm SD & $\begin{array}{c}\text { Pearson } \\
\text { Correlation } \\
\text { Sig. (2-tailed) }\end{array}$ & r-value \\
\hline CTA & $1.845-5.293$ & $3.752 \pm 1.062$ & \multirow{3}{*}{0.006} & \multirow{3}{*}{0.695} \\
\hline & & & & \\
\hline DSA & $1.758-5.488$ & $3.659 \pm 1.251$ & & \\
\hline
\end{tabular}


Table 6. Percentage difference in fistula diameter of ICA on CTA examination to DSA

\begin{tabular}{lccc}
\hline Variable & Mean & Standard Deviation & Median \\
\hline Percentage difference CTA to DSA (\%) & $15.06 \%$ & $14.98 \%$ & $9.75 \%$ \\
\hline
\end{tabular}

Table 7. Interval of the percentage difference of the ICA fistula diameter on CTA to DSA

\begin{tabular}{lll}
\hline Interval & $\mathbf{n}$ & Percentage (\%) \\
\hline $0-20 \%$ & 11 & 78.06 \\
$21-40 \%$ & 2 & 14.03 \\
$>40 \%$ & 1 & 07.01 \\
Total & 14 & 100.0 \\
\hline
\end{tabular}

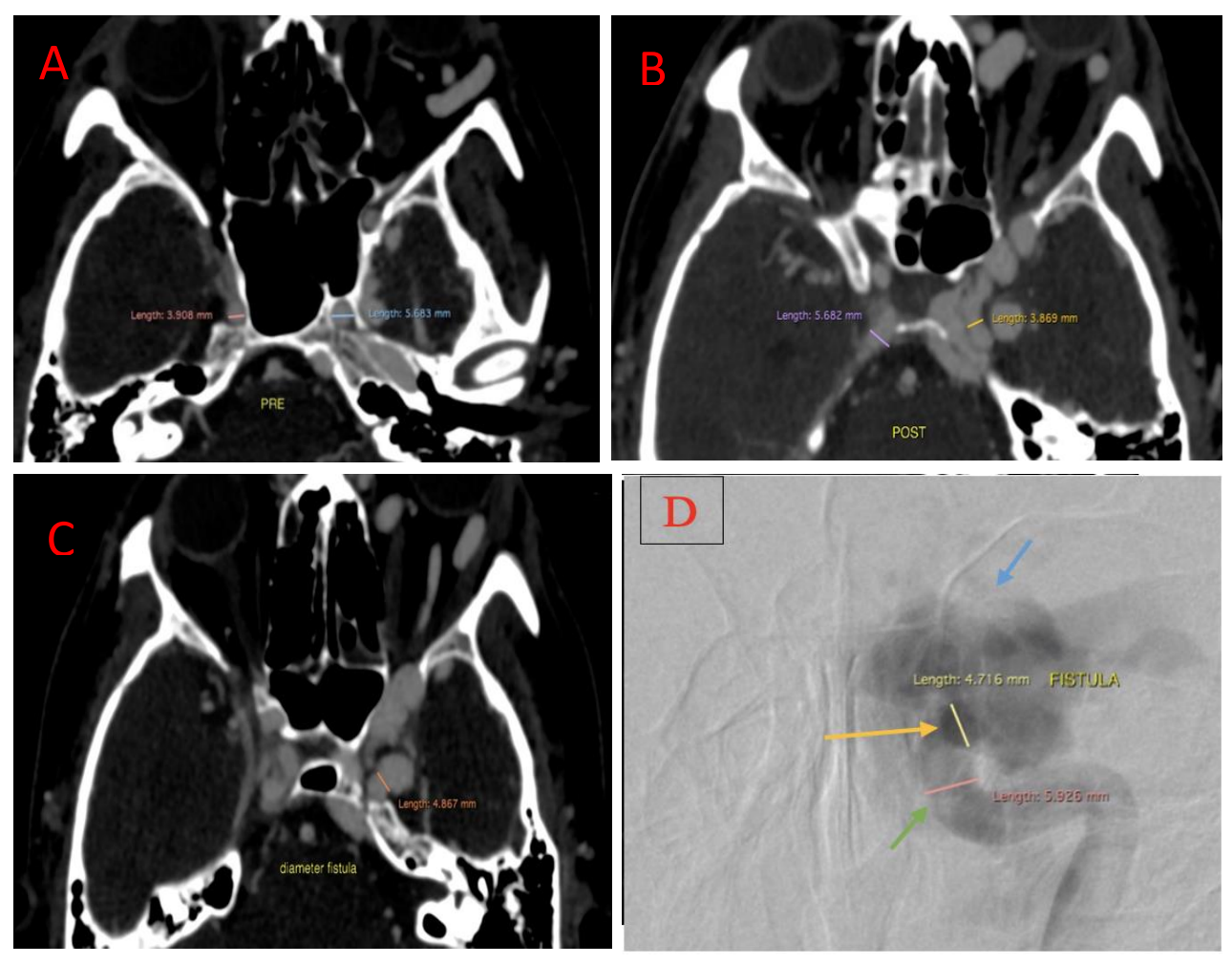

Figure. 2 Axial slices of CTA and DSA (AP view) of the 22-years-old male subject show a left-sided direct CCF (A, B, $\mathrm{C}$, and D). (D) DSA showed a large fistula with a diameter of $4.716 \mathrm{~mm}$ (orange arrow). ICA diameter pre fistula was enlarged (green arrow). The post fistula diameter of the ICA was difficult to evaluate due to no distal flow in ICA (blue arrow) 
Table 8. ICA diameter pre and post fistula in DSA compared to fistula diameter in DSA

\begin{tabular}{lcc}
\hline Variable & Maximum & Minimum \\
\hline Fistula diameter $(\mathrm{mm})$ & 1.758 & 5.488 \\
Comparison between pre and post fistula ICA diameter (mm) & 01.21 & 1.879 \\
Total & & 10 \\
\hline
\end{tabular}

Table 9. Spearman rho test on the comparison of ICA diameter pre and post fistula on CTA and DSA

\begin{tabular}{lll}
\hline Variable & p-value & r value \\
\hline Comparison of ICA diameter pre and post fistula in CTA $\leftrightarrows$ & \\
Comparison of pre and post fistula ICA diameter in DSA & 0.031 & 0.483 \\
\hline
\end{tabular}

\section{Discussion}

In this study, the CCF case was mainly found in the age group > 41 years. Under the CTA examination, there were $53.8 \%$ direct $\mathrm{CCF}$ and $46.1 \%$ indirect $\mathrm{CCF}$. Meanwhile, the DSA examination found $57.7 \%$ direct CCF and 42.3\% indirect CCF. In 2014, El-Hindy et al. reported that direct $\mathrm{CCF}$ is the most common $\mathrm{CCF}$ type A, about $75 \%-80 \%$ overall. This type is often caused by the rupture of the carotid artery wall caused by trauma or a ruptured ICA aneurysm.11

DSA examination showed direct CCF was found at the age of 21 - 40 years (66.7\%) and mostly occurred in males (38.4\%), while indirect CCF was mainly found at age $>41$ years $(61.5 \%)$ and female $(26.9 \%)$. Santos et al. (2014) stated that direct CCF incidence was more in adult males aged 20-30 years, while indirect CCF was more common in women in the age group between the fifth and sixth decades of life. ${ }^{12}$

Patients with suspected CCF require head imaging involving a CTA examination. This study reported that CTA obtained high scores in diagnosing the type of $\mathrm{CCF}$, with a sensitivity value of $93.3 \%$, specificity of $100 \%$, positive predictive value of $100 \%$, negative predictive value of $91.7 \%$, and accuracy $96.15 \%$. This value is not much different from a previous retrospective study by Chen et al. (2005) in CCF patients who undergone DSA and CTA, where CTA had a sensitivity of $87 \%$ compared to a DSA sensitivity of 94.4\%.10 This high diagnostic value proves that CTA can provide information on the type of CCF accurately. As a result, CTA can be trusted in determining the diagnosis and treatment management. CTA is a noninvasive examination, easy to perform, and more acceptable to patients.

Among of 26 cases of CCF, fifteen people with direct CCF based on the DSA examination were measured the fistula diameter. The results of CTA and DSA measurements obtained a value range of $1.845-5.293$ $\mathrm{mm}$ and 1.758 - $5.488 \mathrm{~mm}$, respectively. In this observation, one person showed false negativity on CTA and was diagnosed with the indirect type, then confirmed as the direct type on DSA with a diameter of $1.955 \mathrm{~mm}$ fistula. The results of this study are consistent with various literature, including Korkmazer et al. (2013), which explains that fistula flow varies in direct CCF with high flow shunts, usually measuring between 1- $5 \mathrm{~mm}$ (average $3 \mathrm{~mm}$ ) and depending on the size of the ostium and venous drainage of direct fistula. Whereas indirect CCF, which is a low flow shunt, is difficult to evaluate because it comes from dural bifurcation, which usually has multiple dural feeders and many micro fistulas in the walls of the cavernous sinuses. 13 Lubicz et al. (2007) stated that vascular lesions ${ }^{3} 3 \mathrm{~mm}$ (96.4 - 100\%) showed relatively high 
sensitivity, while lesions $£ 3 \mathrm{~mm}$ showed low sensitivity $(63.6-81.8 \%) .{ }^{14}$

Statistical analysis Pearson correlation test (2tailed) showed a significant relationship between the two variables with a strong criterion relationship $(\mathrm{p}=$ 0.006 and $r$ value $=69.5 \%$ ). Langer et al. (2004) reported a high-flow variant of CCF with 100\% CTA sensitivity and had a high correlation with angiography to visualize the extent of the lesion, presence of corticalvenous drainage, and the location and morphology of the nidus. 9

The difference in the percentage of fistula diameter between CTA and DSA examination of direct CCF was $1.22 \%$ to $48.40 \%$. The percentage difference in the interval shows that $78 \%$ (11 people) have a $0-20 \%$ difference to DSA. No similar studies and literature describe the percentage difference in fistula diameter measurements on CTA and DSA. However, the result of this percentage difference measurement shows that CTA has an accuracy value close to DSA as the standard for the initial diagnosis of $\mathrm{CCF}$, identification of flow thus it can determine the selection of embolization (Chen et al., 2005). 10

The fistula diameter in 15 subjects of the direct CCF according to the DSA examination was $1.758-5.488$ $\mathrm{mm}$, while the ratio between the ICA pre and post fistula diameters was 1.210-1.879 mm. Five post fistula ICA diameters cannot be evaluated due to high flow (large diameter fistula), resulting in all the flow into the fistula so that there is no or no visible flow in the post fistula (distal ICA). Based on observations with DSA, this evaluation dominantly shows the consistency that the larger the fistula diameter, the greater the ratio between the ICA pre fistula diameter; thus, the post fistula diameter gets smaller. In 2018, Henderson \& Miller said angiographically, rapid filling into the cavernous sinus at high flow CCF resulted in reduced or no flow to the intracranial vascular system. In contrast, there was a slow filling of the cerebral venous system through the fistula in low flow fistulas while maintaining flow filling-intracranial arteries. ${ }^{15}$ Besides, Ramuzat et al., 2003 conducted a study on the steal phenomenon in the radiochepalic arteriovenous fistula, explaining that the flow in the brachial artery occurs if a fistula occurs, and the proximal radial artery will increase and cause a decrease in flow in the distal radial artery. 16

Correlation test using the Spearman rho test on comparing the pre and post fistula ICA diameter sizes in both CTA and DSA obtained a significance value of 0.031 with an r-value of $4.83 \%$. That there is a significant relationship with the level of moderate relationship strength. There is no literature or previous research that describes the evaluation between pre and post fistula ICA diameter in CTA to DSA. However, based on these results, CTA supports diameter assessment and can also be used as an initial reference in identifying high flow and low flow lesions in CCF.

This study has several limitations: 1) This study is a retrospective study in a short period dan the total sample is relatively small. 2) Placement of the measurement location has a high subjectivity factor, and this difference creates bias. 3) The fistula diameter measured on the CTA and DSA examinations does not directly reflect the size of the fistula because some fistulas overlap by contrast which causes the measurement results to be different.

\section{Conclusion}

The results of the comparison of the types of $\mathrm{CCF}$ on the CTA and DSA examination were very strong, and the level of similarity of CTA and DSA was $92.2 \%$. It could be concluded that there was a significant relationship between CTA and DSA.

The evaluation of the comparison of the pre and post fistula ICA diameter in DSA dominantly shows that the larger the ICA pre fistula diameter, the smaller the ICA post fistula diameter due to all flow is into the fistula on the DSA examination. The correlation of the ICA fistula diameter measurement results on CTA to DSA was strong, while the relationship of pre and postfistula ICA diameter in CTA to DSA was moderate.

We hope that a similar study will be carried out comparing the contralateral to ipsilateral vascular involvement of the $\mathrm{CCF}$ lesions to complement the results of this study and assist the Interventional 
Radiologists in preparing and carrying out follow-up investigation in the future.

\section{Acknowledgment}

I would like to express my deepest gratitude to all radiology teaching staff at Faculty of Medicine Universitas Airlangga, my family, and my friends for their support.

\section{References}

1. De Keizer RJW. Carotid-cavernous and orbital arteriovenous fistulas: Ocular features, diagnostic and hemodynamic considerations in relation to visual impairment and morbidity. Orbit. 2003;22(2):121-42.

2. Luo CB, Teng MMH, Chang FC, Chang CY. Traumatic indirect carotid cavernous fistulas: angioarchitectures and results of transarterial embolization by liquid adhesives in 11 patients. Surg Neurol [Internet]. 2009;71(2):216-22. Available from: http:/ /dx.doi.org/10.1016/j.surneu.2007.0 9.032

3. Ellis, J. A., Goldstein, H., Connolly, E. S., \& Meyers PM. Carotid-cavernous fistulas. Neurosurg Focus [Internet]. 2012;32(5):E9. Available from: http:/ / thejns.org/doi/abs/10.3171/2012.2. FOCUS 1223

4. Riskesdas K. Hasil Utama Riset Kesehata Dasar (RISKESDAS). J Phys A Math Theor [Internet]. 2018;44(8):1-200. Available from: http: / /arxiv.org/abs / 1011.1669\%OAhttp:/ / dx.doi.org/10.1088/1751-

8113/44/8/085201\%0Ahttp:/ /stacks.iop.or g/ $1751-$

$8121 / 44 / \mathrm{i}=8 / \mathrm{a}=085201$ ?key=crossref.abc 74 c979a75846b3de48a5587bf708f

5. Romijn M, Gratama Van Andel HAF, Van Walderveen MA, Sprengers ME, Van Rijn JC, Van Rooij WJ, et al. Diagnostic accuracy of CT angiography with matched mask bone elimination for detection of intracranial aneurysms: Comparison with digital subtraction angiography and 3D rotational angiography. Am J Neuroradiol. 2008;29(1):134-9.

6. Latchaw RE, Alberts $\mathrm{MJ}$, Lev $\mathrm{MH}$, Connors JJ, Harbaugh RE, Higashida RT, et al. Recommendations for imaging of acute ischemic stroke: A scientific statement from the american heart association. Stroke. 2009;40(11):3646-78.

7. Laviña B. Brain vascular imaging techniques. Int J Mol Sci. 2017;18(1).

8. Yamamoto M, Okura $\mathrm{Y}$, Ishihara $\mathrm{M}$, Kagemoto M, Harada K, Ishida T. Development of digital subtraction angiography for coronary artery. J Digit Imaging. 2009;22(3):319-25.

9. Langer PD, Reed MD, Turbin RE, Farkas JA. Diagnosis and Evaluation of Carotid Cavernous Fistulas and Arterio-Venous Malformation of the Cavernous Sinus using CT Angiography (CTA). Invest Ophthalmol Vis Sci. 2004;45(13):257.

10. Chen CCC, Chang PCT, Shy CG, Chen WS, Hung HC. CT angiography and MR angiography in the evaluation of carotid cavernous sinus fistula prior to embolization: A comparison of techniques. Am $\mathrm{J}$ Neuroradiol. 2005;26(9):2349-56.

11. El-Hindy N, Kalantzis G, Patankar T, Georgalas I, Jyothi S, Goddard T, et al. Difficult indirect carotid-cavernous fistulasalternative techniques to gaining access for treatment. Clin Interv Aging. 2014;9:168790.

12. Santos D dos, Monsignore LM, Nakiri GS, Cruz AAV e, Colli BO, Abud DG. Diagnóstico por imagem das fístulas arteriovenosas da região do seio cavernoso. Radiol Bras. 2014;47(4):251-5.

13. Korkmazer B, Kocak B, Tureci E, Islak C, Kocer N, Kizilkilic O. Endovascular treatment of carotid cavernous sinus fistula: A 
systematic review. World J Radiol. 2013;5(4):143.

14. Lubicz B, Levivier M, François O, Thoma P, Sadeghi N, Collignon L, et al. Sixty-four-row multisection CT angiography for detection and evaluation of ruptured intracranial aneurysms: Interobserver and intertechnique reproducibility. Am J Neuroradiol. 2007;28(10):1949-55.

15. Henderson AD, Miller NR. Carotid-cavernous fistula: Current concepts in aetiology, investigation, and management. Eye [Internet]. 2018;32(2):164-72. Available from: http: / dx.doi.org/10.1038/eye.2017.240

16. Ramuzat A, How T V., Bakran A. Steal phenomenon in radiocephalic arteriovenous fistula. In vitro haemodynamic and electrical resistance simulation studies. Eur J Vasc Endovasc Surg. 2003;25(3):246-53. 